\title{
Neuroprotective effect of matrine on MPTP-induced Parkinson's disease and on Nrf2 expression
}

\author{
FANHUA MENG $^{1,2}$, JIANHUI WANG ${ }^{2}$, FUXIANG DING ${ }^{3}$, YUNLIANG XIE ${ }^{4}$, YINGJIE ZHANG ${ }^{4}$ and JIE ZHU $^{1}$ \\ ${ }^{1}$ Department of Neurology, The First Hospital, Jilin University, Changchun, Jilin 130021; ${ }^{2}$ Neurology Department 1; \\ ${ }^{3}$ Cardiovascular Department 1; ${ }^{4}$ Neurology Department 2, The Affiliated Hospital of Beihua University, \\ Jilin 132011, P.R. China
}

Received July 14, 2015; Accepted September 2, 2016

DOI: $10.3892 / \mathrm{ol} .2016 .5383$

\begin{abstract}
The incidence rate of Parkinson's disease (PD) is $\leq 2 \%$ in Chinese individuals $>65$ years old, accounting for $40 \%$ of the global total of PD patients. The pathogenesis of PD is not yet clear, and oxidative stress-induced mitochondrial dysfunction is considered to be the main reason for the onset of PD. Studies have shown that matrine exhibits good antioxidant activity. Thus, the present study aimed to observe the protective effect and mechanism of matrine on 1-methyl-4-phenyl-1,2,3,6-tetrahydropyridine (MPTP)-induced dopaminergic neuron damage. A total of 25 C57BL male mice were randomly divided into 5 groups, consisting of the control group (group A), the MPTP group (group B) and three matrine (4, 8 and $16 \mathrm{mg} / \mathrm{kg}$ ) plus MPTP treatment groups (groups C, D and E, respectively). Results from a pole-climbing test and locomotor activity experiments were recorded. The mice were sacrificed 4 days later and brain dissection was performed. The levels of superoxide dismutase (SOD) and glutathione (GSH) were assessed. The expression level of tyrosine hydroxylase (TH) in the ventral midbrain was studied by immunofluorescence analysis. The expression level of nuclear factor erythroid 2-related factor 2 (Nrf2) in the ventral midbrain was studied by western blot analysis. The experiments were repeated three times. Compared with control mice, the PD mice exhibited the typical behaviors associated with PD; matrine can alleviate this phenomenon, and with increasing matrine concentration, the symptoms
\end{abstract}

Correspondence to: Mr. Yingjie Zhang, Neurology Department 2, The Affiliated Hospital of Beihua University, 12 Jiefang Zhong Road, Jilin 132011, P.R. China

E-mail: xieyunliang0630@163.com

Mr. Jie Zhu, Department of Neurology, The First Hospital, Jilin University, 71 Xinmin Street, Changchun, Jilin 130021, P. R. China E-mail: jiezhu0573@sina.com

Key words: matrine, Parkinson's disease, dopamine neurons, nuclear factor erythroid 2-related factor 2, 1-methyl-4-phenyl-1,2,3,6-tetrahydropyridine were reduced significantly. Compared with the control mice, the PD mice had lower SOD and GSH activity, and matrine partially reversed the change in SOD and GSH activity. Immunofluorescence analysis showed that the level of TH in the ventral midbrain decreased significantly in the PD mice, and that the mice administered matrine showed higher expression of TH and levels of TH-positive cells. Western blotting results showed that the expression of Nrf2 in the ventral midbrain decreased significantly in the PD mice, and that matrine was able to reverse this phenomenon. In conclusion, by promoting antioxidant-related Nrf2 signaling pathways in the ventral midbrain, matrine can inhibit the oxidative damage of dopamine neurons in PD.

\section{Introduction}

Oxidative stress is involved in the development and progression of various neurodegenerative diseases, including Parkinson's disease (PD), Alzheimer's disease and amyotrophic lateral sclerosis (1). PD is a progressive neurodegenerative disease with an unknown pathogenesis, and the loss of substantia nigra dopaminergic neurons is characteristic of its lesions (2-5). Oxidative stress-induced mitochondrial dysfunction is hypothesized to be the main reason for the pathogenesis of PD (6). After neurotoxin 1-methyl-4-phenyl-1,2,3,6-tetrahydropyridine (MPTP) induction, PD can be modeled in vitro. MPTP can cross the blood-brain barrier, and is converted into its active metabolite methyl-4-phenylpyridine (MPP+), which due to affinity with the dopamine transporter, is thus selectively transported to the mitochondria of the dopaminergic neurons. As a neurotoxic metabolite, MPP+ can block cell respiration and promote active oxygen [reactive oxygen species (ROS) formation], thus inducing the death of dopaminergic neurons (7).

While traditional levodopa replacement therapy and certain non-steroidal anti-inflammatory drugs can reduce the clinical symptoms, they do not prevent progression of the disease and long-term medication can cause serious adverse reactions $(8,9)$. Matrine can be obtained from plants of the Sophora genus and has always been used in traditional Chinese medicine to treat inflammation (10). Matrine has been shown to produce a wide range of pharmacological effects 
and has been used to treat a variety of diseases, including viral hepatitis, neuropathic pain and isoproterenol-induced heart disease (11-13). In addition, significant antitumor effects have been found in gastric cancer, rhabdomyosarcoma, acute myeloid leukemia and breast cancer $(14,15)$, and studies have shown that matrine exhibits antioxidant effects in a number of diseases. PD is mainly caused by damage to dopamine neurons, and oxidative stress is one of its important pathogenetic factors. There is little literature on the interaction between matrine and the MPTP-induced damage to mouse dopaminergic neurons in PD. Accordingly, the present study investigated whether matrine has a protective effect on dopaminergic neurons, and the viral mechanisms involved were studied.

\section{Materials and methods}

Materials. C57BL, 7 to 8-month-old, male mice (weighing 20-25 g) were purchased from Beijing Vital River Laboratory Animal Technology Co., Ltd. (Beijing, China). The mice were housed in a thermostatically controlled environment with set lighting conditions (lighting time, 7:30 a.m. to 7:30 p.m.). A total of 25 mice were randomly divided into five groups, namely the control group (group A), the MPTP group (group B) and three matrine $(4,8$ and $16 \mathrm{mg} / \mathrm{kg}$ ) plus MPTP treatment groups (groups C, D and E, respectively). The control group received saline by intraperitoneal injection (30 mg/kg/day for 4 days), and the MPTP group was continuously administered an intraperitoneal injection of $30 \mathrm{mg} / \mathrm{kg}$ MPTP for 4 days (once a day) to create the PD mouse model. The matrine + MPTP groups were treated with different doses of matrine $(4,8$ and $16 \mathrm{mg} / \mathrm{kg}$ ) in advance, $8 \mathrm{~h}$ prior to intraperitoneal injection with MPTP.

The study was approved by the Ethics Committee of the College of Basic Medical Sciences, Jilin University (Changchun, Jilin, China).

Equipment, drugs and reagents. An ultra-pure water system (Milli-Q Synthesis) was purchased from Millipore (Darmstadt, Germany) and an automatic embedding machine (model no. EG-1140C) was purchased from Leica Microsystems, Inc. (Buffalo Grove, IL, USA). A slicing machine (model no. X-202A) was purchased from Guangdong Yi Mai Technology Co., Ltd. (Guangdon, China), an inverted phase contrast microscope was obtained from Olympus Corporation (model no. BX51), and constant current regulator electrophoresis (model no. DYC-40C) and semi-dry transfer membrane (model no. DYY-8B) instruments were purchased from Beijing Liuyi Biotechnology Co., Ltd. (Beijing, China). Matrine (catalog no. CDS016735), MPTP (catalog no. M0896) and rabbit anti-mouse tyrosine hydroxylase (TH) antibody (catalog no. T8700) were purchased from Sigma-Aldrich (Millipore). Rabbit anti-mouse nuclear factor E2-related factor 2 (Nrf2; catalog no. 12721P) and rabbit anti-mouse $\beta$-actin (catalog no. 12620; dilution, 1:10,000) antibodies were purchased from Cell Signaling Technology, Inc. (Danvers, MA, USA). The concentrated DAB kit was purchased from Zhongshan Golden Bridge Biotechnology Co., Ltd. (Beijing, China; catalog no. ZLI-9017), the superoxide dismutase (SOD) test kit (catalog no. A001-3) and the glutathione (GSH) test kit (catalog no. A006) were purchased from Nanjing Jiancheng Bioengineering Institute (Nanjing, Jiangsu, China), and the CytoBuster protein extraction reagent was purchased from Novagen Inc. (Madison, WI, USA; catalog no. 71009-3).

Establishment and execution of a mouse model of PD. Under a constant temperature and lighting conditions (lighting time, 7:30 a.m. to 7:30 p.m.), the mice were continuously treated for 4 days, and suspension and climbing experiments were conducted every day. On the fourth day, all mice were decapitated, then the mouse brain striatum and substantia nigra were isolated; one portion was used for protein extraction, and the other portion was stored at room temperature after being embedded in paraffin.

Suspension experiment. The C57BL mice were placed on a horizontal wire of $\sim 1.5 \mathrm{~mm}$ in diameter, suspended $30 \mathrm{~cm}$ from the ground, and the hang time was recorded to detect mouse limb coordination. Scoring criteria: 0-5 sec, 0 points; 6-10 sec 2 points; $11-15 \mathrm{sec}, 3$ points; $16-20 \mathrm{sec}, 4$ points; and $>20 \mathrm{sec}, 5$ points.

Pole-climbing test. A tube of $\sim 30 \mathrm{~cm}$ in length and $\sim 1 \mathrm{~cm}$ in diameter was gauze wrapped and a wooden ball was attached to the top. Mice were placed on top of the wooden ball and the time required for the mouse to traverse from the top to the bottom of the tube was recorded. Time were compared prior to and after PD modeling.

SOD and GSH detection. The levels of SOD and GSH were detected according to the manufacturer's instructions provided in the SOD (catalog no. A001-3) and GSH (catalog no. A006) test kits (Nanjing Jiancheng Bioengineering Institute).

TH antigen test. The paraffin sections were made into frozen sections and dried at room temperature for $15 \mathrm{~min}$, the stained tissue was marked by Pap Pen, soaked for $10 \mathrm{~min}$ in PBS to remove the OCT and then film-fixed at room temperature for $30 \mathrm{~min}$ with $4 \%$ paraformaldehyde. The sections were treated for 10 min with $2.2 \%$ Triton X-100, and then washed with PBS twice and blocked with $10 \%$ normal goat serum in PBS at room temperature for $1 \mathrm{~h}$. TH antibody was added (1:3,000 dilution) and the section was incubated overnight at $4^{\circ} \mathrm{C}$, followed by being washed with PBS twice. The Alexa Fluor 488-labeled goat anti-rabbit immunoglobulin G secondary antibody was added and the section was incubated at $37^{\circ} \mathrm{C}$ for $1 \mathrm{~h}$ prior to being washed again with PBS twice. Fluorescence microscopy was performed on five randomly selected fields, and the number of positive cells was counted and analyzed statistically.

Nigrostriatal protein extracts. The nigrostriatal tissue was homogenized, then washed twice with ice-cold PBS and centrifuged at $400 \mathrm{x} g$ for $5 \mathrm{~min}$. The supernatant was discarded, and the $100 \mathrm{X}$ protease inhibitors and CytoBuster protein extraction reagent were added to the precipitate, pipetted and mixed at room temperature for $15 \mathrm{~min}$, prior to centrifugation at $12,000 \mathrm{x} \mathrm{g}$ for $15 \mathrm{~min}$ at $4^{\circ} \mathrm{C}$. The resulting supernatant contained the desired protein. 


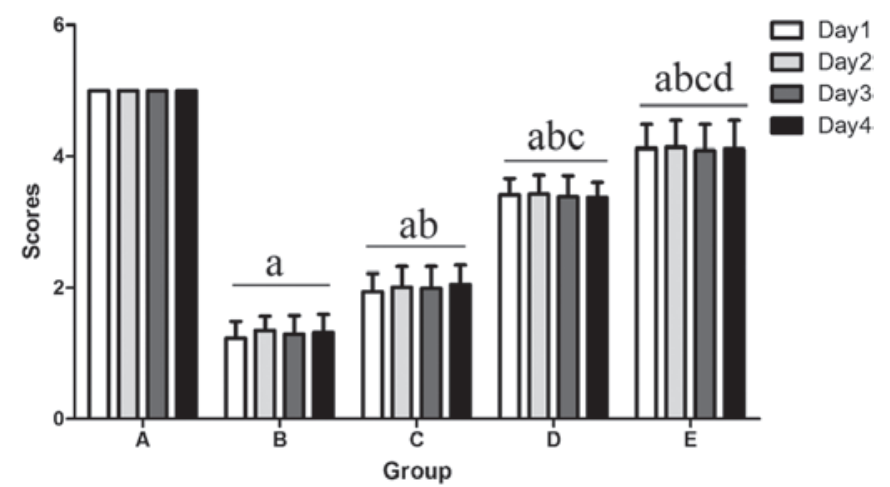

Figure 1. Effects of matrine on the suspension of mice with 1-methyl-4-phenyl-1,2,3,6-tetrahydropyridine-induced Parkinson's disease. Increasing suspension ability score was observed with increasing matrine concentration. ${ }^{\mathrm{a}} \mathrm{P}<0.01$ vs. group $\mathrm{A} ;{ }^{\mathrm{b}} \mathrm{P}<0.01$ vs. group $\mathrm{B}$; ${ }^{\mathrm{c}} \mathrm{P}<0.01$ vs. group $\mathrm{C}$; and ${ }^{\mathrm{d}} \mathrm{P}<0.01$ vs. group $\mathrm{D}$.

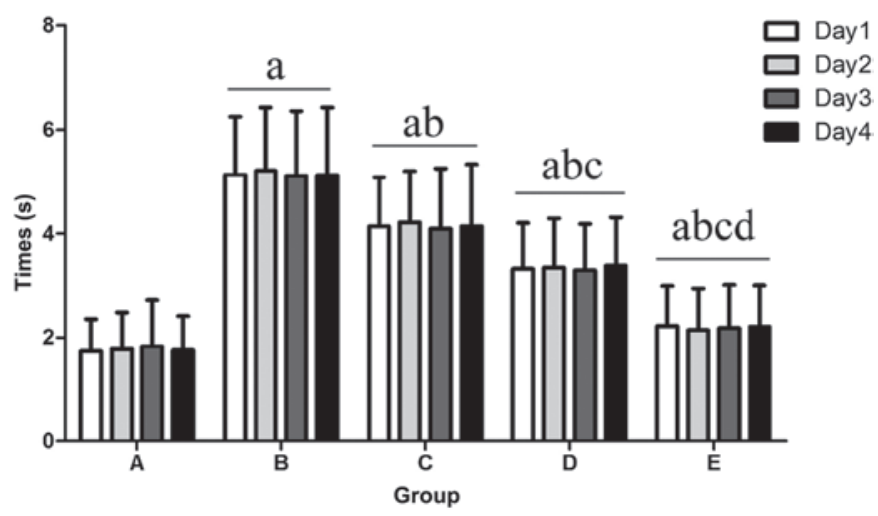

Figure 2. Effect of matrine on the pole-climbing ability of mice with 1-methyl-4-phenyl-1,2,3,6-tetrahydropyridine-induced Parkinson's disease. Increasing pole-climbing ability recovery was observed with increasing matrine concentration. ${ }^{\mathrm{a}} \mathrm{P}<0.01$ vs. group $\mathrm{A} ;{ }^{b} \mathrm{P}<0.01$ vs. group $\mathrm{B}$; ${ }^{\mathrm{c}} \mathrm{P}<0.01$ vs. group $\mathrm{C}$; and ${ }^{\mathrm{d}} \mathrm{P}<0.01$ vs. group $\mathrm{D}$.

Western blotting. The extracted protein was isolated using $10 \%$ SDS-PAGE gel separation and a 5\% stacking gel, and then semi-transferred to nitrocellulose membranes using incubation in TBST containing 5\% BSA at room temperature for 2 h. Nrf2 rabbit anti-mouse antibodies (1:1,000 dilution)were added and the membranes were incubated at $4^{\circ} \mathrm{C}$ overnight. The next day, the membrane was washed with $0.1 \%$ TBST 3 times, for $5 \mathrm{~min}$ each, and the horseradish peroxidase (HRP)-labeled goat anti-rabbit secondary antibody was added for incubation at room temperature for $1 \mathrm{~h} .0 .1 \%$ TBST was used to wash the membrane, using Supersignal West Femto/Pico HRP-sensitive chemiluminescent substrate for coloration. Rabbit anti-mouse $\beta$-actin (1:10,000 dilution) was used as an internal control. All experiments were repeated at least 3 times.

Statistical. Using SPSS 15.0 statistical software (SPSS, Inc., Chicago, IL, USA), multiple sets of data were compared using a one-way analysis of variance, and differences between two groups of data were compared using the Student-Newman-Keuls analysis method. All data are presented as the mean \pm standard deviation. $\mathrm{P}<0.05$ was considered to indicate a statistically significant difference.

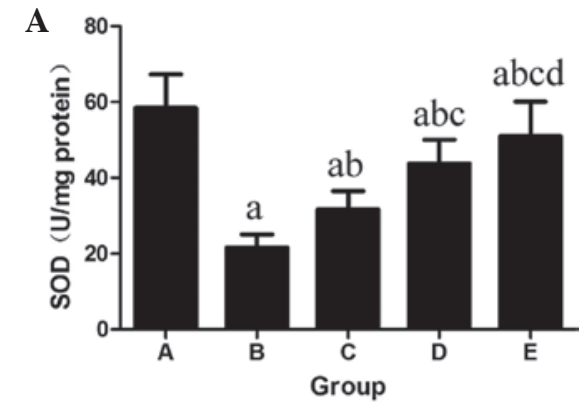

B

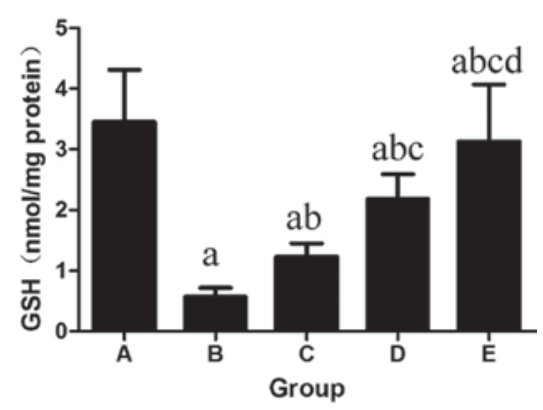

Figure 3. Effect of matrine on SOD and GSH content in mouse brain tissue. Increasing brain activity recovery was observed with increasing matrine concentration. ${ }^{\mathrm{a}} \mathrm{P}<0.01$ vs. group $\mathrm{A} ;{ }^{\mathrm{b}} \mathrm{P}<0.01$ vs. group $\mathrm{B}$; ${ }^{\mathrm{c}} \mathrm{P}<0.01$ vs. group $\mathrm{C}$; and ${ }^{\mathrm{d}} \mathrm{P}<0.01$ vs. group D. SOD, superoxide dismutase; GSH, glutathione.

\section{Results}

Effect of matrine on the suspension ability of mice with PD. The control mice exhibited a mean suspension ability score of 5. Compared with the control group, the mean score in the MPTP mice was significantly lower $(\mathrm{P}<0.001)$, while matrine administration significantly alleviated this phenomenon $(\mathrm{P}=0.004)$. The suspension ability score increased with increasing matrine concentration $(\mathrm{P}<0.001)$ (Fig. 1).

Effect of matrine on pole-climbing ability. Compared with the control group, MPTP mice exhibited a significantly reduced capacity for pole-climbing, with a significantly longer climb time $(\mathrm{P}<0.001)$. The administration of matrine was able to significantly alleviate this phenomenon $(\mathrm{P}=0.008)$, and the pole-climbing ability recovered more significantly with increasing matrine concentration $(\mathrm{P}<0.001)$ (Fig. 2).

Mouse brain tissue SOD and GSH assay. Compared with the control group, the MPTP mice exhibited significantly lower SOD and GSH activity in the brain tissues $(\mathrm{P}<0.001)$, while the administration of matrine significantly alleviated this phenomenon $(\mathrm{P}=0.006)$. The mouse brain activity recovered more significantly with increasing matrine concentration $(\mathrm{P}<0.001)$ (Fig. 3).

Immunohistochemistry. In the comparison of dopamine $\mathrm{TH}$ expression in the substantia nigra and the striatum, relative to the control group, the number of $\mathrm{TH}$-positive cells of the MPTP group significantly decreased $(\mathrm{P}<0.001)$. In the matrine-treated mice, a higher expression level of $\mathrm{TH}$ and a greater number of TH-positive cells was evident (Fig. 4). 
Group

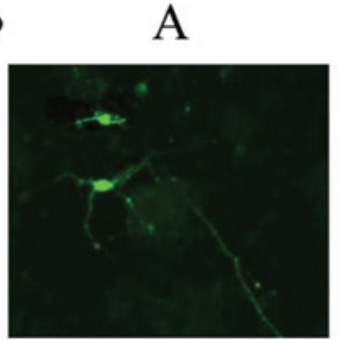

B

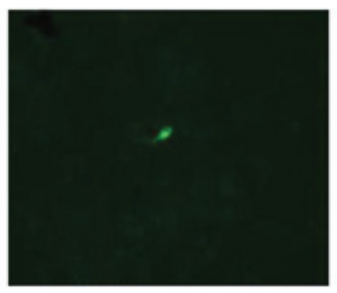

$\mathrm{C}$

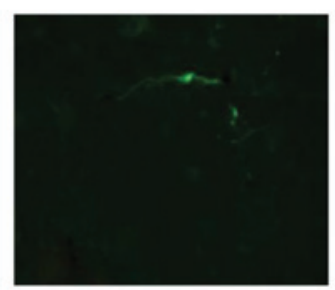

$\mathrm{D}$

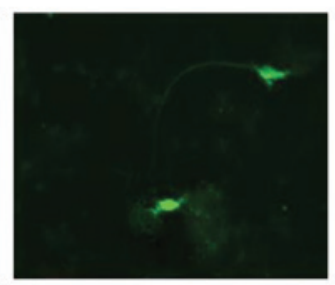

$\mathrm{E}$

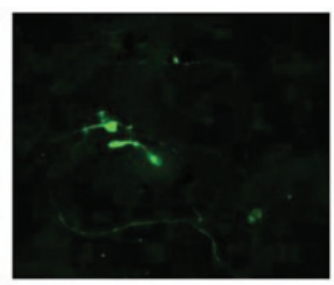

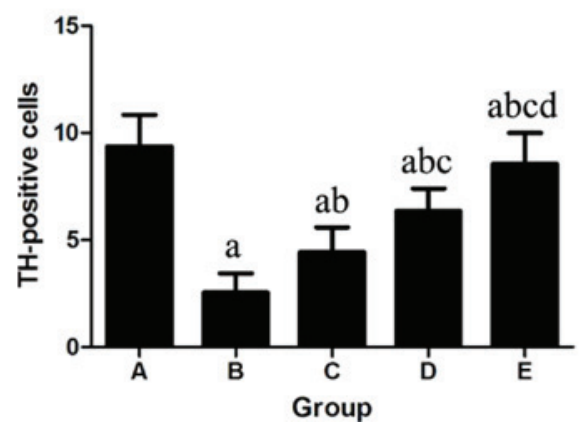

Figure 4. Immunohistochemical detection of the antigen expression of the substantia nigra and striatal $\mathrm{TH}$. ${ }^{\mathrm{a}} \mathrm{P}<0.01$ vs. group $\mathrm{A} ;{ }^{b} \mathrm{P}<0.01 \mathrm{vs}$. group $\mathrm{B} ;{ }^{\mathrm{c}} \mathrm{P}<0.01$ vs. group $\mathrm{C}$; and ${ }^{\mathrm{d}} \mathrm{P}<0.01$ vs. group $\mathrm{D}$.
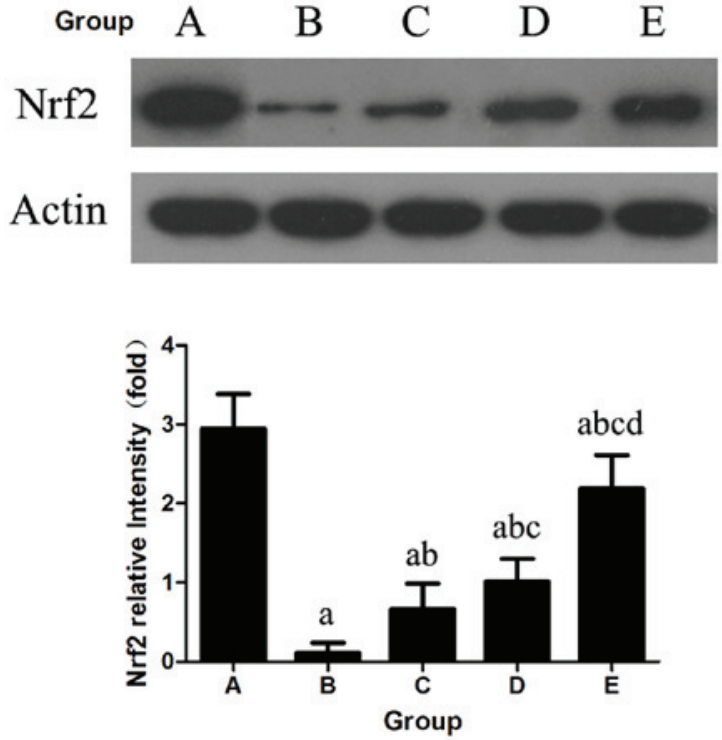

Figure 5. Analysis of the expression of Nrf2 of Western blot. Compared with group $\mathrm{A},{ }^{\mathrm{a}} \mathrm{P}<0.01$, compared with group $\mathrm{B},{ }^{\mathrm{b}} \mathrm{P}<0.01$, compared with group $\mathrm{C}$, ${ }^{\mathrm{C}} \mathrm{P}<0.01$, compared with group $\mathrm{D},{ }^{\mathrm{d}} \mathrm{P}<0.01$.

Western blot analysis detecting the expression of Nrf2. The relative intensity of antioxidant-related $\mathrm{Nrf} 2$ molecules decreased significantly in the MPTP group, while in the matrine-treated mice, stronger Nrf2 expression was exhibited in the substantia nigra and striatum, which increased in a concentration-dependent manner (Fig. 5).

\section{Discussion}

PD is a progressive neurodegenerative disease whose main symptoms include bradykinesia, resting tremors and rigidity (16-18). In the present study, using an MPTP-induced mouse PD model, reduced motion bradykinesia, which is typical of PD, was apparent along with other symptoms of the disease. Oxidative stress is the cause of a number of neurodegenerative diseases, including PD (6). Due to a high metabolic rate, brain tissue is prone to hypoxia, and this may lead to an increase in reactive oxygen species (ROS) and oxidative stress. SOD is one of the key enzymes in the oxidative stress defense and is key to good health (19). GSH, formed from a combination of glutamic acid, cysteine and glycine, is a tripeptide containing a thiol group, and has antioxidant and detoxification integration (20). The activity of SOD and GSH may reflect the free radical scavenging ability of the body.

When PD occurs, brain tissue SOD and GSH activity is significantly reduced. In the present study, SOD and GSH activity was significantly reduced in the cerebral tissue of the MPTP mice. In addition, immunofluorescence showed a significant reduction in the TH expression of MPTP mice at the substantia nigra and the striatum. The described animal model meets the requirements established for PD in this study.

Matrine obtained from plants of the Sophora genus has always been used in traditional Chinese medicine to treat inflammation (10). Studies have shown that matrine can prevent the steatohepatitis caused by a high carbohydrate diet (21) through its antioxidant effects. PD is mainly caused by a loss of dopamine neurons, and oxidative stress is important in the pathogenesis. Little literature is available on whether matrine can protect dopamine neurons in mice with MPTP-induced PD and the specific mechanisms involved. The results of the present study showed that following the administration of matrine treatment, a number of the typical symptoms of PD significantly improved, SOD and GSH activity appeared significantly increased in the brain tissue, and a higher level of $\mathrm{TH}$ expression and more TH-positive cells were evident. This suggests that matrine may have a significant therapeutic effect on PD. 
Nrf2 can regulate the expression of 200 genes, including a number of antioxidant genes (22). Nrf2 protects against the islet $\beta$ cell damage caused by acute oxidative stress (23). The present study further examined the matrine treatment of PD by regulating the expression of Nrf2 to investigate how matrine protect dopamine neurons. The results showed that Nrf2 expression in the MPTP group was significantly decreased, while the administration of matrine resulted in stronger Nrf2 expression in the substantia nigra and the striatum, in a concentration-dependent manner. In summary, the results of the present study confirmed that matrine exhibited a significant therapeutic effect in mice with PD and demonstrated that the mechanism of matrine treatment in PD may be the inhibition of oxidative damage of dopamine neurons by the promotion of antioxidant-related Nrf2 signaling pathways.

\section{References}

1. Barnham KJ, Masters CL and Bush AI: Neurodegenerative diseases and oxidative stress. Nat Rev Drug Discov 3: 205-214, 2004.

2. Wood JM and Gupta S: Vitamin D and neurocognitive disorder due to Alzheimer's disease: A review of the literature. Ann Clin Psychiatry 27: e1-e7, 2015.

3. Sargolzaei S, Sargolzaei A, Cabrerizo M, Chen G, Goryawala M, Noei S, Zhou Q, Duara R, Barker W and Adjouadi M: A practical guideline for intracranial volume estimation in patients with Alzheimer's disease. BMC Bioinformatics 16 (Suppl 7): S8, 2015

4. Zhang C, Hu WH, Wu L, Zhang K and Zhang JG: Behavioral effects of deep brain stimulation of the anterior nucleus of thalamus, entorhinal cortex and fornix in a rat model of Alzheimer's disease. Chin Med J (Engl) 128: 1190-1195, 2015.

5. Herman Lara H, Alanís-Garza EJ, Estrada Puente MF, Mureyko LL, Alarcón Torres DA and Ixtepan Turrent L: Nutritional approaches to modulate oxidative stress that induce Alzheimer's disease. Nutritional approaches to prevent Alzheimer's disease. Gac Med Mex 151: 245-251, 2015 (In Spanish).

6. Langston JW and Irwin I: MPTP: Current concepts and controversies. Clin Neuropharmacol 9: 485-507, 1986.

7. Alcaraz-Zubeldia M, Rojas P, Boll C and Rios C: Neuroprotective effect of acute and chronic administration of copper (II) sulfate against MPP+ neurotoxicity in mice. Neurochem Res 26: 59-64, 2001.

8. Yacoubian TA and Standaert DG: Targets for neuroprotection in Parkinson's disease. Biochim Biophys Acta 1792: 676-687, 2009.

9. Samii A, Etminan M, Wiens MO and Jafari S: NSAID use and the risk of Parkinson's disease: Systematic review and meta-analysis of observational studies. Drugs Aging 26: 769-779, 2009.

10. Li Y, Wang B, Zhou C and Bi Y: Matrine induces apoptosis in angiotensin II-stimulated hyperplasia of cardiac fibroblasts: Effects on Bcl-2/Bax expression and caspase-3 activation. Basic Clin Pharmacol Toxicol 101: 1-8, 2007.
11. Long Y,Lin XT, Zeng KL and Zhang L: Efficacy of intramuscular matrine in the treatment of chronic hepatitis B. Hepatobiliary Pancreat Dis Int 3: 69-72, 2004.

12. Haiyan W, Yuxiang L, Linglu D, Tingting X, Yinju H, Hongyan L, Lin M, Yuanxu J, Yanrong W and Jianqiang Y: Antinociceptive effects of matrine on neuropathic pain induced by chronic constriction injury. Pharm Biol 51: 844-850, 2013.

13. Li X, Zhou R, Zheng P, Yan L, Wu Y, Xiao X and Dai G: Cardioprotective effect of matrine on isoproterenol-induced cardiotoxicity in rats. J Pharm Pharmacol 62: 514-520, 2010.

14. Li Y, Zhang J, Ma H, Chen X, Liu T, Jiao Z, He W, Wang F, Liu X and Zeng X: Protective role of autophagy in matrine-induced gastric cancer cell death. Int J Oncol 42: 1417-1426, 2013.

15. Guo L, Xue TY, Xu W and Gao JZ: Effects of matrine on the proliferation and apoptosis of human rhabdomyosarcoma RD cells. Zhongguo Dang Dai Er Ke Za Zhi 14: 780-784, 2012 (In Chinese).

16. Haga R, Sugimoto K, Nishijima H, Miki Y, Suzuki C, Wakabayashi K, Baba M, Yagihashi S and Tomiyama M: Clinical utility of skin biopsy in differentiating between Parkinson's disease and multiple system atrophy. Parkinsons Dis 2015: 167038, 2015.

17. Akbar U, He Y, Dai Y, Hack N, Malaty I, McFarland NR, Hess C, Schmidt P, Wu S and Okun MS: Weight loss and impact on quality of life in Parkinson's disease. PLoS One 10: e0124541, 2015.

18. Liu HG, Zhang K, Yang AC and Zhang JG: Deep brain stimulation of the subthalamic and pedunculopontine nucleus in a patient with Parkinson's disease. J Korean Neurosurg Soc 57: 303-306, 2015.

19. Sun WH, Liu F, Chen, Y and Zhu YC: Hydrogen sulfide decreases the levels of ROS by inhibiting mitochondrial complex IV and increasing SOD activities in cardiomyocytes under ischemia/reperfusion. Biochem Biophys Res Commun 421: 164-169, 2012.

20. Bo Young Chung, So Ra Choi, Ik Jun Moon, et al: The glutathione derivative, GSH monoethyl ester, may effectively whiten skin but GSH does not. Int J Mol Sci 17: E629, 2016.

21. Zhang HF, Shi LJ, Song GY, Cai ZG, Wang C and An RJ: Protective effects of matrine against progression of high-fructose diet-induced steatohepatitis by enhancing antioxidant and anti-inflammatory defences involving Nrf2 translocation. Food Chem Toxicol 55: 70-77, 2013.

22. Abdul-Aziz A, MacEwan DJ, Bowles KM and Rushworth SA: Oxidative stress responses and NRF2 in human leukaemia. Oxid Med Cell Longev 2015: 454659, 2015.

23. Fu J, Zheng H, Wang H, Yang B, Zhao R, Lu C, Liu Z, Hou Y, $\mathrm{Xu}$ Y, Zhang Q, et al: Protective role of nuclear factor E2-related factor 2 against acute oxidative stress-induced pancreatic $\beta$-cell damage. Oxid Med Cell Longev 2015: 639191, 2015. 